\title{
The NASA Advanced Exploration Systems Nuclear Thermal Propulsion Project
}

\author{
Michael G. Houts ${ }^{1}$, Doyce P. Mitchell², Tony Kim³ ${ }^{3}$ William J. Emrich ${ }^{4}$, Robert R. Hickman ${ }^{5}$, Harold P. Gerrish ${ }^{6}$, \\ Glen Doughty ${ }^{7}$, \\ NASA Marshall Space Flight Center, MSFC, AL 35812 \\ Anthony Belvin ${ }^{8}$ \\ US Department of Energy, Washington, D.C. 20585 \\ Steven Clement ${ }^{9}$ \\ US Department of Energy / National Nuclear Security Adminstration, Las Vegas, NV 89193 \\ Stanley K. Borowski ${ }^{10}$ \\ NASA Glenn Research Center, Cleveland, $\mathrm{OH}, 44135$, \\ John Scott ${ }^{11}$ \\ NASA Johnson Space Center, Houston, TX, 77058 \\ and \\ Kevin P. Power ${ }^{12}$ \\ NASA Stennis Space Center, MS, 39529
}

\begin{abstract}
The fundamental capability of Nuclear Thermal Propulsion (NTP) is game changing for space exploration. A first generation NTP system could provide high thrust at a specific impulse ( $\left.I_{s p}\right)$ above $900 \mathrm{~s}$, roughly double that of state of the art chemical engines. Characteristics of fission and NTP indicate that useful first generation systems will provide a foundation for future systems with extremely high performance. The role of a first generation NTP in the development of advanced nuclear propulsion systems could be analogous to the role of the DC-3 in the development of advanced aviation systems.
\end{abstract}

\section{Nomenclature}

CFEET = Compact Fuel Element Environmental Test

DOE $\quad=$ Department of Energy

HAT = NASA Human Architecture Team

HIP $\quad=$ Hot Isostatic Press

\footnotetext{
${ }^{1}$ Nuclear Research Manager, Technology Development and Transfer Office/ZP30, Member

${ }^{4}$ NTREES Lead Engineer, Propulsion Research and Technology Branch/ER24, Member

${ }^{5}$ Assistant Branch Chief, Metal Joining and Processes Branch/EM32, Member

${ }^{6}$ Technical Assistant, Propulsion Systems Design and Integration Division/ER20, Member

${ }^{7}$ Engineer, Propulsion and Research Technology Branch /ER24, Member Department of Energy/NE75

${ }^{9}$ Manager, National Criticality Experiments Research Center (NCERC)/NNSA

${ }^{10}$ Chief, Propulsion and Controls Systems Analysis/86:124, Member

${ }^{11}$ Branch Chief, Direct Energy Conversion,/EP311, Member

12 Project Manager, Engineering Test Directorate/EA61, Member
}

${ }^{2}$ Manager, Nuclear Thermal Propusion Project, Technology Development and Transfer Office/ZP30, Member

${ }^{3}$ Deputy Manager, Nuclear Thermal Propulsion, Technology Development and Transfer Office/ZP30, Member

${ }^{8}$ Program Manager, Space Reactor Research and Development, Office of Space and Defense Power Systems, 
NASA $=$ National Aeronautics and Space Administration

NCPS $=$ Nuclear Cryogenic Propulsion Stage

NTP $=$ Nuclear Thermal Propulsion

NTR $=$ Nuclear Thermal Rocket

NTREES $=$ Nuclear Thermal Rocket Element Environmental Simulator

$P E C \quad=$ Pulsed Electric Current

SLS = Space Launch System

\section{Introduction}

Development efforts in the United States have demonstrated the viability and performance potential of NTP systems. For example, Project Rover (1955-1973) completed 22 high power reactor and fuel tests. Peak performances included operating at a fuel element hydrogen exhaust temperature of $2550 \mathrm{~K}$ and a peak fuel power density of $5200 \mathrm{MW} / \mathrm{m}^{3}$ (Pewee test), operating at a thrust of $930 \mathrm{kN}$ (Phoebus-2A test), and operating individual fule element for an accumulated time of 109 minutes (NF-1 test). ${ }^{1}$ Results from Project Rover indicated that an NTP system with a high thrust-to-weight ratio and a specific impulse greater than $900 \mathrm{~s}$ would be feasible. Excellent results have also been obtained by Russia. Ternary carbide fuels developed in Russia may have the potential for providing even higher specific impulses. Cermet fuels, developed primarily for use in high performance space fission power systems, also show potential to enable high thrust, high $\mathrm{I}_{\mathrm{sp}}$ NTP systems.

Many factors would affect the development of a $21^{\text {st }}$ century nuclear thermal rocket (NTR). Test facilities built in the US during Project Rover are no longer available. However, advances in analytical techniques, the ability to utilize or adapt existing facilities and infrastructure, precision manufacturing, and the ability to develop a limited number of new test facilities may enable a viable development, qualification, and acceptance testing strategy for NTP.

NTP will only be utilized if it is affordable. Testing programs must be optimized to obtain all required data while minimizing cost through a combination of non-nuclear and nuclear testing. Strategies must be developed for affordably completing required nuclear testing. A schematic of an NTP engine is shown in Figure 1.

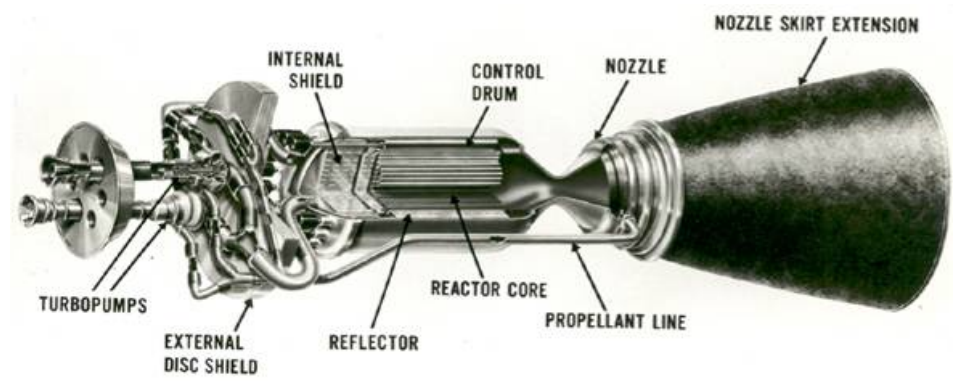

Figure 1. Schematic of an NTP Engine

\section{The Nuclear Thermal Propulsion Project}

The precursor to the NASA Advanced Exploration Systems (AES) NTP project was the Nuclear Cryogenic Propulsion Stage (NCPS) project. Initiated in October, 2011, the goal of that project was to assess the affordability and viability of an NCPS. Key elements of that project included: 1) Pre-conceptual design of the NCPS and architecture integration; 2) Development of a High Power ( 1 MW input) Nuclear Thermal Rocket Element Environmental Simulator (NTREES); 3) NCPS Fuel Design and Testing; 4) NCPS Fuels Testing in NTREES and the Compact Fuel Element Environmental Test (CFEET) system; and 5) Affordable NCPS Development and Qualification Strategy. The NCPS project involved a large ( $\sim 50$ person) NASA/DOE team supplemented with a small amount of procurement funding for hardware and experiments. In addition to evaluating fundamental technologies, the team assessed many aspects of the integrated NCPS, and its applicability to enable NASA architectures of interest.

Under the AES NTP project, a "leader/follower" fuel selection has been made for a potential flight demonstration engine using highly enriched uranium (HEU) fuel. The fuel recommendation was made by an independent review panel (IRP) consisting of 8 highly qualified experts who were chartered to recommend a fuel assuming the use of HEU and the desire for an early flight demonstration of NTP. Although work will continue 
related to both fuels, the AES NTP project will focus on a uranium bearing graphite composite "lead" fuel and on HEU engine designs that could potentially utilize that fuel. In addition, the AES NTP project is focusing on a more detailed assessment of operational facilities within the Department of Energy (DOE), NASA, industry, and academia that could help enable affordable development and utilization of NTP.

\section{A. Pre-Conceptual Design of a NTP Engine and Architecture Integration}

NTP is an in-space propulsion system/stage using fission as the energy source to heat propellant (hydrogen) and expand it though a nozzle to create thrust. The increase in engine performance available from even a first generation NTP system would enable ambitious exploration missions, both robotic and human. It is the intent of the AES NTP project to develop a pre-conceptual design of a first generation stage with one or more nuclear thermal rocket(s) capable of interfacing with soon to be available launch vehicles and possible payloads and missions. The design must utilize technologies that are readily available with minimal risk to development. The design must take into account the development viability/feasibility, affordability, and safety. If possible, work should be focused on engine designs and thrust levels that would be directly applicable to supporting a human Mars mission. Other strategic considerations are an affordable testing approach and the need for sustained funding.

NTP must show relevance to U.S. space exploration goals and must provide a development path toward a feasible, affordable, and sustainable NTP system. United States' National Space Policy (June 28, 2010, pg. 11) specifies that NASA shall: By 2025, begin crewed missions beyond the Moon, including sending humans to an asteroid. By the mid-2030s, send humans to orbit Mars and return them safely to Earth. NTP system design will focus on ensuring maximum benefit to human Mars mission, although NTP could have numerous other applications as well.

NTP mission analysis and definition will stay synchronized with the NASA Human Architecture Team (HAT) for application toward future human Mars missions and the currently developing Space Launch System (SLS). The AES NTP project will provide input to SLS future block upgrades to enhance efficiency for volume and mass constraints that will reduce the number of launches for a mission utilizing NTP.

NTP system trades and analysis will naturally optimize the system to accomplish human Mars missions. The sensitivity of stage performance to specific impulse, engine thrust-to-weight ratio, and other parameters will be assessed to accomplish the mission and eventually define the actual size and weight of the system. The design of the NTP system will favor proven and tested technologies and will identify critical technologies that will be required for development.

A historical perspective for a common, scalable fuel element will help provide flexibility in design. During the Rover program, a common fuel element / tie tube design was developed and used in the 50 klbf Kiwi-B4E (1964), 75 klbf Phoebus-1B (1967), 250 klbf Phoebus-2A (June 1968), then reduced to the 25 klbf Pewee engine (Nov-Dec 1968).

NASA and DOE are investigating a similar approach: design, build, ground- and then flight-test a small affordable engine that, if clustered, can be used for human missions. This appropriate sized NTP engine could provide significant benefits to a wide variety of other potential exploration missions.

The stage will need to leverage technologies from other programs and projects. Some specific technologies that are important to NTP are listed below:

o Cryogenic Storage (long duration storage, cryo-coolers, zero boil-off, zero leakage)

o Automated rendezvous \& docking

o Radiation hardening of electronics

o Radiation shielding

O Enhanced System Health and Status Sensor/ Post operation/test remote inspection evaluation

NTP should take maximum advantage of technologies, components, and subsystems that are developed elsewhere in the architecture, as well as provide input and requirements to those technologies to obtain the capabilities needed for effective integration of NTP. The AES NTP project should also stay connected to the SLS and upper Cryogenic Propulsion Stage (CPS) projects to take advantage of common elements and to leverage technologies and configurations to reduce cost.

To support the NTP design effort, available analytical tools would be enhanced and refined. The DOE has developed sophisticated computational modeling tools for nuclear system design. Since the initial fuel elements under consideration are very similar to the past work accomplished under the Rover/NERVA and other programs, NTP would be able to take advantage of these available models. NASA has many rocket system simulation tools. These computational modeling tools from DOE and NASA would be used in conjunction to respond quickly to needed trade studies and mission analyses. 
One engine system model under consideration is the closed expander cycle, which derives fluid-pumping power from heat generated within the engine and passes the entire propellant flow through the nozzle. The cycle is currently of interest due to its high $\mathrm{I}_{\mathrm{sp}}$ performance. Also, hydrogen is the most desirable propellant based on its thermodynamic properties; similarly for high $\mathrm{I}_{\text {sp }}$ performance. Liquid hydrogen also has a very low density and high volume tanks are advantageous for many missions.

The safety of all rocket engines (including nuclear engines) is paramount. Although a nuclear engine is essentially non-radioactive prior to operation at significant power, the engine must be designed to avoid an inadvertent start. This is particularly true for times when individuals could be in close proximity to the reactor, such as launch processing. Safety of the nuclear engine will be ensured via design and by leveraging seven decades of reactor operating experience.

Crew health and safety may benefit from the use of NTP. NTP may enable shorter mission times (reducing crew exposure to microgravity, cosmic rays, solar flares, and other hazards) or increased payload mass (allowing for increased shielding, supplies, or equipment.

\section{B. Development of a High Power ( 1 MW input) Nuclear Thermal Rocket Element Environmental Simulator}

A high temperature, high power density fissile fuel form is a key technology for NTP. Fuel life and performance is largely limited by mass loss in a hot gas/cyclic environment. Hence, a major milestone of the NTP project is the completion and initial utilization of the 1-MW Nuclear Thermal Rocket Element Environmental Simulator (NTREES) test chamber. The purpose of the NTREES facility (which also includes an arc heater and the Compact Fuel Element Environmental Tester, CFEET) is to perform realistic non-nuclear testing of nuclear thermal rocket (NTR) fuel elements and fuel materials. Although the NTREES facility cannot mimic the neutron and gamma environment of an operating NTR, it can simulate the thermal hydraulic environment within an NTR fuel element to provide critical information on material performance and compatibility. The NTREES upgrade has progressed to the point that it is now capable of testing uranium-bearing fuel elements.

Once fully operational, the 1-MW NTREES test chamber will be capable of testing fuel elements and materials in flowing hydrogen at pressures up to $1000 \mathrm{psi}$, at temperatures up to and beyond $3000 \mathrm{~K}$, and at near-prototypic reactor channel power densities. NTREES will be capable of testing potential fuel elements with a variety of propellants, including hydrogen with additives to inhibit corrosion of certain potential NTR fuel forms; however the current focus remains on pure hydrogen propellants.

The NTREES facility is licensed by the Nuclear Regulatory Commission (NRC) to test fuels containing depleted uranium. It includes a pyrometer suite to measure fuel temperature profiles and a mass spectrometer to help assess fuel performance and evaluate potential material loss from the fuel element during testing. Additional diagnostic upgrades planned for NTREES include the addition of a gamma ray spectrometer located near the vent filter to detect uranium fuel particles exiting the fuel element in the propellant exhaust stream and to provide additional information of any material loss occurring during testing. Using propellant fed from gas storage trailers located external to the facility, NTREES is configured to allow continuous, uninterrupted testing of fuel elements for any desired length of time. A picture of the current NTREES primary chamber configuration is shown in Figure 2.

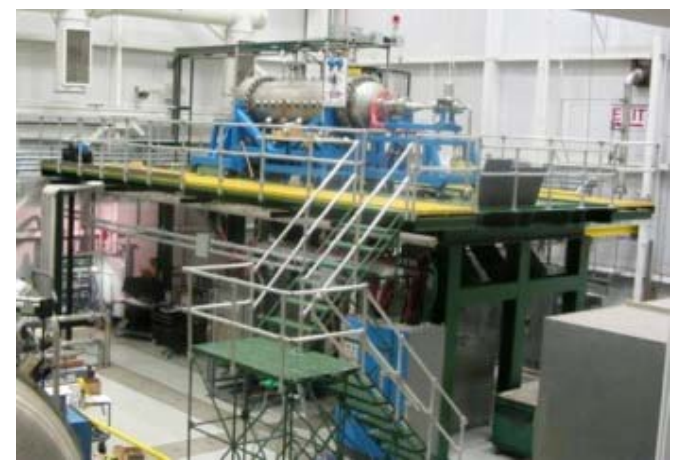

Figure 2. Nuclear Thermal Rocket Element Environmental Simulator.

An additional test facility associated with NTREES is an operational arc heater (Figure 3) that is capable of flowing hot hydrogen over a material or fuel sample at a hydrogen gas temperature of up to $3160 \mathrm{~K}$ for 
approximately 30 minutes. This facility could be used for the preliminary vetting of material samples. Also available is CFEET, capable of testing small fuel samples at high temperatures in a hydrogen environment (Figure 4).

CFEET testing has been particularly useful in the development of fuel elements with the desired microstructure and properties. The smaller fuel element segments used in CFEET testing save both time and schedule due to reduced depleted uranium requirements and other factors.

This project will also develop a detailed understanding of the energy deposition and heat transfer processes in NTREES, along with effects on material mechanics and fluid/material interaction, to better improve future test conditions and obtain as much information as possible to accurately extrapolate non-nuclear test data to real reactor conditions.

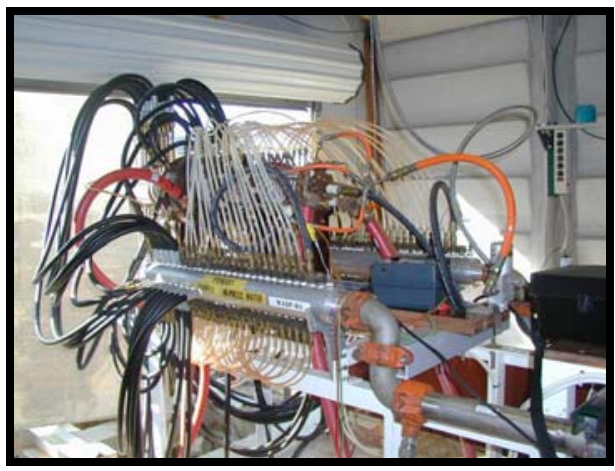

Figure 3. Arc Heater.

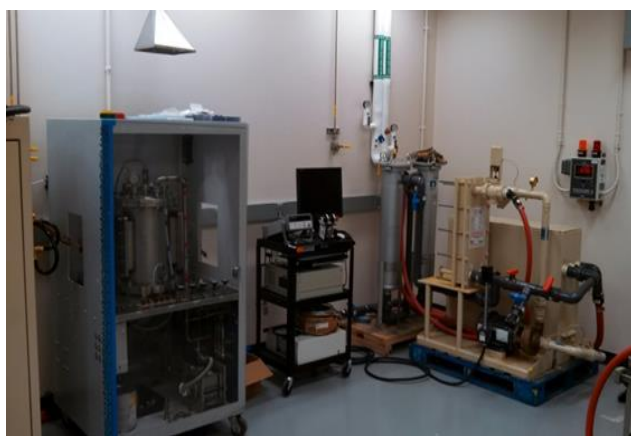

Figure 4. Compact Fuel Element Environmental Test facility (CFEET)

\section{NTP Fuel Design / Fabrication}

Early fuel materials development is necessary to validate requirements and minimize technical, cost, and schedule risks for future exploration programs. The development of a stable fuel material is a critical path, long lead activity that will require a considerable fraction of program resources. The objective of the NTP Fuel Design and Fabrication task is to demonstrate materials and process technologies for manufacturing robust, full-scale CERMET and graphite composite fuel elements. The elements will be based on the starting materials, compositions, microstructures, and fuel forms that were demonstrated in previous programs. The development will be a phased approach to recapture key technologies and produce quality fuels. Samples will then be tested in flowing hot hydrogen to understand processing and performance relationships. As part of this demonstration task, a final full scale element test will be performed to validate robust designs. These demonstrations are necessary to enable a future fuel materials downselection process. A major focus of the NTP project is the use of a highly integrated NASA/DOE/Industry/Academia fuels development team. The goal is to enhance and utilize existing infrastructure and capabilities to minimize cost.

Current research at MSFC and the Center for Space Nuclear Research (CSNR) at INL is focused on developing fabrication processes for prototypical $\mathrm{W} / \mathrm{UO}_{2}$ cermet fuel elements. Cermets are typically formed by densification of powders using Powder Metallurgy (PM) processes. Tungsten based CERMETS with surrogate ceramic particles have been fabricated to near theoretical density using Hot Isostatic Press (HIP) and Pulsed Electric Current (PEC) techniques. During HIP, the CERMET powders are consolidated in sacrificial containers at $2000^{\circ} \mathrm{C}$ and pressures up to $30 \mathrm{ksi}$. The PEC process consists of high speed consolidation of powders using DC current and graphite dies. For both HIP and PEC processing, the powder size and shape, powder loading, and processing parameters significantly affect the quality and repeatability of the final part. Figure 5 shows a typical microstructure and image of a net shape consolidated CERMET part. The part is a 19 hole configuration that had uniform shrinkage during consolidation and good tolerance on the flow channel geometry. 

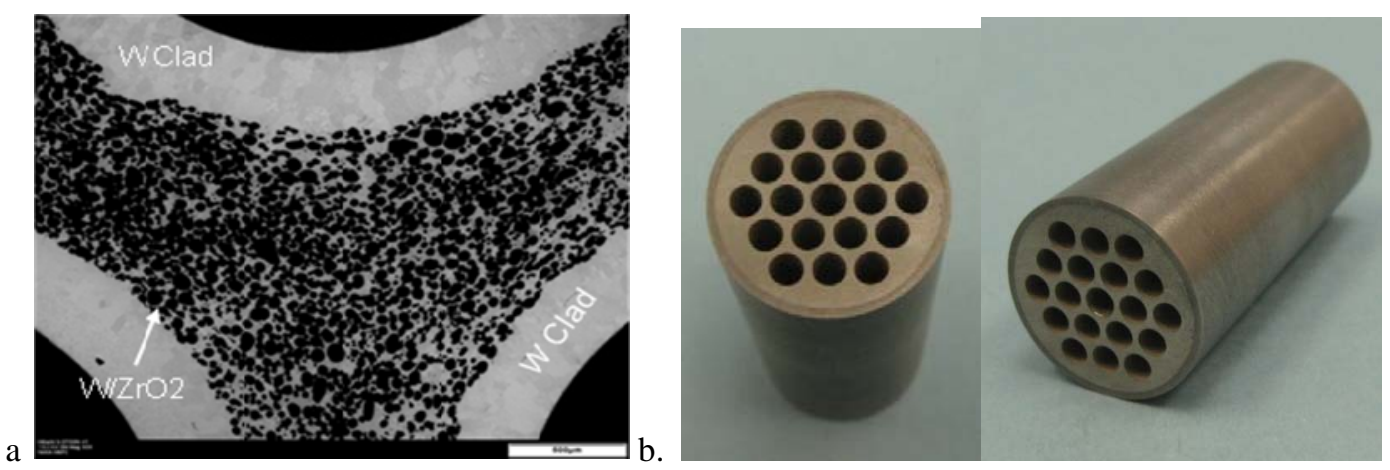

Figure 5. a) Micrograph of a W/60 vol\% $\mathrm{ZrO}_{2}$ CERMET with integral $\mathrm{W}$ claddings b) Consolidated W/40 vol\% HfN CERMET sample.

The nature of this initial task is rapid materials and process screening as a precursor to the detailed development that will be required to fully optimize and qualify a cermet fuel. Cermet materials and processes were demonstrated at subscale level on previous efforts, but there are significant technical and programmatic challenges for key technologies. Some of the materials and process approaches being developed to maximize performance are the size of the fuel particles and resultant shape in the consolidated part, CVD tungsten coating of spherical $\mathrm{UO}_{2}$ particles prior to consolidation, complete surface cladding of the elements with tungsten, and additions of small amounts of fuel particle and matrix stabilization materials such as $\mathrm{Gd}_{2} \mathrm{O}_{3}$.

Significant work is also being done at ORNL to recapture graphite composite fuel materials tested in the NF-1 experiment at the end of the Rover/NERVA program. Various graphite based fuels consisting of $\mathrm{UO}_{2}, \mathrm{UC}_{2}$, or (U, $\mathrm{Zr}$ )C particles in a graphite matrix were tested in the Rover/NERVA program. Many of the materials were successfully demonstrated in full scale nuclear test engines. However, the fuel materials and fabrication technologies are not currently available. The NTP task is focused on recapturing the graphite composite extrusion and $\operatorname{ZrC}$ coating capabilities. The composite fuel matrix is a carbide-based ceramic fuel composition consisting of uranium carbide, zirconium carbide and graphite materials. Subscale matrix samples are being fabricated and tested to demonstrate microstructure and properties. In parallel, coating trials are being performed on short elements for hot hydrogen testing at MSFC. The goal is to partially validate recapture of coated graphite composite fuel element technology by testing a 16" segment of an element with Rover/NERVA geometry in the NTREES. Figure 6 shows a recent graphite composite extrusion performed at ORNL. Recent pogress includes extrusion (at ORNL) of a 16”, 4-hole graphite composite surrogate (hafnium) bearing fuel element that was then sent to MSFC for high

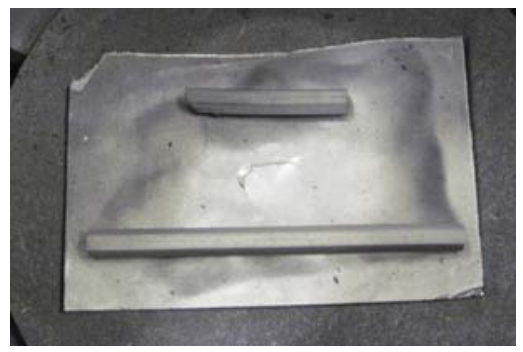

Figure 6: Graphite composite fuel element extrusion at ORNL

temperature heat treatment. The surrogate fuel element will serve as a pathfinder for a possible test of a graphite composite fuel element with representative depleted uranium loading. Testing of the surrogate fuel element in NTREES is anticpated to occur in 2015.

\section{NTP Fuels Testing in NTREES}

Testing in NTREES will range from fuel sample testing using CFEET to the testing of near-prototypic fuel elements. A primary goal of the testing is to demonstrate adequate fuel performance and to increase confidence in fuel system designs (e.g. materials, coatings, geometries) prior to potential nuclear testing. CERMET and graphite 
composite samples will be thermal cycle tested in a static and flowing environment. Several iterations of testing will be performed to evaluate fuel mass loss impacts from density, microstructure, fuel particle size and shape, chemistry, claddings, particle coatings, and stabilizers. Initial subscale testing is being performed in the CFEET system. The CFEET test samples are typically 0.75 " across the flats and up to 3 " long for solid slug and prototypic 7 or 19-hole channel configurations. The 7 or 19-hole channel configuration is being used for CFEET screening to rapidly evaluate thermal cyclic affects on prototypic geometries from surface vaporization, diffusion/migration, and cracking. Testing has shown that fuel mass loss is significantly impacted by thermal cycling and geometry. The prototypical geometry will be much more susceptible to cracking induced migration and volatilization of the exposed fuel particles. The fuel materials and forms such as coated particles, claddings, and stabilizers being evaluated on this effort have all been demonstrated to control fuel migration and loss. The initial screening is not to determine or characterize specific modes of fuel loss or mechanisms. The intent is to verify performance improvements of the materials and processes prior to expensive full scale fabrication and testing. Posttest analysis includes weight percent fuel loss, microscopy (SEM, EBSD, and EDS), and dimensional tolerance and cracking.

Subsequent testing of full scale fuel elements will be performed in NTREES. The test samples will be based on the Rover/NERVA and ANL 200MW designs. The goal is to benchmark performance in NTREES for comparison

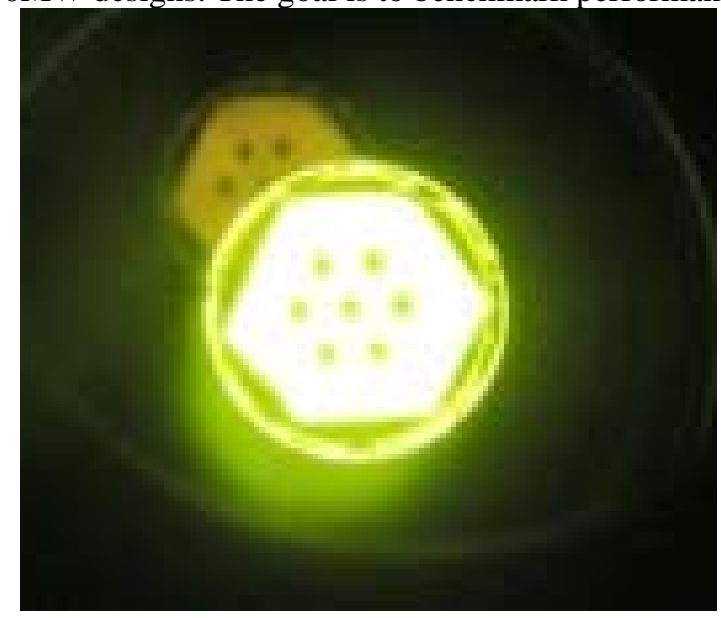

Figure 7. W / UO2 Sample Undergoing Testing in CFEET

to future materials and process improvements, alternate fabrication processes, and other fuel materials of interest. The iterative materials and process development, CFEET screening, and NTREES testing is anticipated to continue into FY 2015 and beyond. A photograph of a W / UO2 CERMET sample undergoing testing in CFEET is shown in Figure 7.

\section{E. Affordable NTP Development and Qualification Strategy}

As previously noted, both the US and Russia have conducted highly successful NTR ground test and technology development programs. Although all of those programs were cancelled prior to flight, the cancellation typically occurred because the mission requiring NTP was cancelled, not because of insurmountable issues associated with the NTR. However, if NTP is to be used, its development, qualification, and utilization must be affordable and done in a way that is technically, programmatically, and politically acceptable.

Lessons learned have also been acquired from the J-2X rocket engine program, ARES 1-X Test Flight Program, and X-43A Flight Demo Program. The major factors from the lessons learned include the following: Follow NASA standards unless deviation has concurrence from the chief engineer and safety officer, start with low safety factors and evolve, upfront involvement from Safety Mission Assurance (including Risk Management) and Systems Engineering Integration, test development engines to the extremes and test two certification engines for flight with double the burn duration and double the number of starts.

\section{Conclusion}

The potential capability of NTP is game changing for space exploration. A first generation NTP system could provide high thrust at a specific impulse above $900 \mathrm{~s}$, roughly double that of state of the art chemical engines. Near- 
term NTP systems would provide a foundation for the development of significantly more advanced, higher performance systems. For NTP to be utilized, an affordable development and qualification strategy must be devised.

\section{References}

${ }^{1}$ Koenig D. R., “Experience Gained from the Space Nuclear Rocket Program (Rover),” LA-10062-H, Los Alamos National Laboratory, Los Alamos, NM, 1986. 